\title{
Mobility-based SIR model for complex networks: with case study Of COVID-19
}

\author{
Rahul Goel $^{1}$ (D) - Loïc Bonnetain ${ }^{2} \cdot$ Rajesh Sharma $^{1} \cdot$ Angelo Furno $^{2}$
}

Received: 12 April 2021 / Revised: 15 July 2021 / Accepted: 6 August 2021 / Published online: 22 October 2021

(c) The Author(s), under exclusive licence to Springer-Verlag GmbH Austria, part of Springer Nature 2021

\begin{abstract}
In the last decade, humanity has faced many different pandemics such as SARS, H1N1, and presently novel coronavirus (COVID-19). On one side, scientists have developed vaccinations, and on the other side, there is a need to propose models that can help in understanding the spread of these pandemics as it can help governmental and other concerned agencies to be well prepared, especially for pandemics, which spreads faster like COVID-19. The main reason for some epidemic turning into pandemics is the connectivity among different regions of the world, which makes it easier to affect a wider geographical area, often worldwide. Also, the population distribution and social coherence in the different regions of the world are non-uniform. Thus, once the epidemic enters a region, then the local population distribution plays an important role. Inspired by these ideas, we propose two versions of our mobility-based SIR model, (i) fully mixed and (ii) for complex networks, which especially takes into account real-life interactions. To the best of our knowledge, this model is the first of its kind, which takes into account the population distribution, connectivity of different geographic locations across the globe, and individuals' network connectivity information. In addition to presenting the mathematical proof of our models, we have performed extensive simulations using synthetic data to demonstrate the generalization capability of our models. Finally, to demonstrate the wider scope of our model, we applied our model to forecast the COVID-19 cases at county level (Estonia) and regional level (Rhône-Alpes region in France).
\end{abstract}

Keywords COVID-19 $\cdot$ Epidemic based modeling $\cdot$ SIR $\cdot$ Mobility $\cdot$ Complex networks $\cdot$ Call data records $\cdot$ Estonia $\cdot$ Rhône-Alpes

\section{Introduction}

In this modern age, pandemics are not a rare phenomenon. As in the last decade, we have seen several pandemics such as H1N1, SARS, and EBOLA, and presently humanity is facing its biggest crisis due to COVID-19. The severity of

Rahul Goel

Rahul.Goel@ut.ee

Loïc Bonnetain

loic.bonnetain@entpe.fr

Rajesh Sharma

Rajesh.Sharma@ut.ee

Angelo Furno

Angelo.Furno@univ-eiffel.fr

1 Institute of Computer Science, University of Tartu, Tartu, Estonia

2 LICIT UMR_T9401, University of Lyon, ENTPE, University Gustave Eiffel, Lyon, France these pandemics can be understood by the death toll claimed by them. According to WHO, the pandemic H1N1/09 virus resulted in 18,036 deaths (Organization WH et al 2009). On the other hand, the Centers for Disease Control and Prevention (CDC) estimate between 151,700 and 575,400 deaths due to the pandemic H1N1/09 virus (Centers for Disease Control and Prevention 2012). Currently, the coronavirus (COVID-19) pandemic, which started in December 2019 from Wuhan, China, has infected 134,641,198 individuals and claimed 2,917,995 (as of April 9,2021 ) deaths worldwide (CSSE 2020) COVID. Pandemics are different from epidemics in terms of their geographic spread. An epidemic affects many people at the same time. It spreads from person to person and remains local to a specific region. In comparison, when an epidemic engulfs an entire country, continent, or the whole world, it is termed as pandemic.

In the past, various models have been proposed for understanding the epidemics spread. These models can be broadly classified into two categories, that is agent-based modeling 


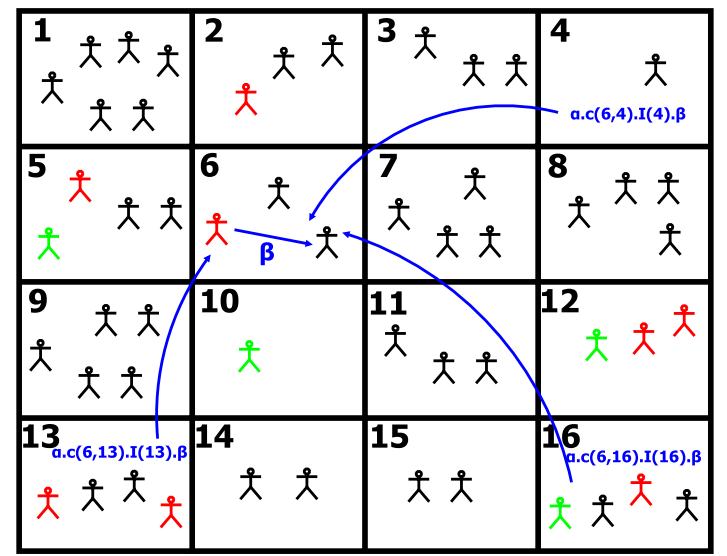

(a) Fully-Mixed Model

Fig. 1 Local And Global Transmission Of Infection In Fully Mixed and Complex Networks Model: Each cell represents a separate region with some population density (here regions are 1 to 16). Individuals in each cell are color-coded: Black (Susceptible), Red (Infected), and Green (Recovered). The local transmission rate of infection is $\beta$ for all cells. a shows the fully mixed model and infection in this model can transfer as follows: For region 6, its social connectivity is $\alpha$. The mobility of individuals from region 4 to region 6 and fraction of infected individuals at region 4 is represented as $c(6,4)$ and

(Bonabeau 2002; Schelling 1971; Sun 2006) and compartmental models (Kermack and McKendrick 1927; Hethcote 2000; Goel et al. 2019). The agent-based modeling is used for simulating the actions and interactions of autonomous agents as a whole (Epstein 2009). These agents can be both individual or collective entities such as organizations or groups. In contrast, differential equations are used in compartmental models, where the population is divided into different compartments such as suspected (S), infected (I), and recovered (R) (Kermack and McKendrick 1927). Several other variants of these models have also been proposed such as SI (Hurley et al. 2006), SIS (Nåsell 1996), SIR (Kermack and McKendrick 1927), and SIRS (Jin et al. 2007).

Agent-based model researchers often criticize compartmental models as these models struggle to capture the connectivity between different regions of the globe, and different real-world population characteristics, such as worldwide population distribution (Chinazzi et al. 2020; Eubank et al. 2004). In this study, we propose a mobility-based SIR model, an extension to the classical SIR-based epidemic model, which considers the real-world population distribution across different regions of the world. Most importantly, the model also takes into account the connectivity factor among various regions over the world, which is the key cause in accelerating the process of transforming epidemics into pandemics. We present two versions of our model, (i) fully mixed and (ii) complex networks model, which especially takes into account real-life interactions. We model the regions in a 2-dimensional lattice, where each cell represents

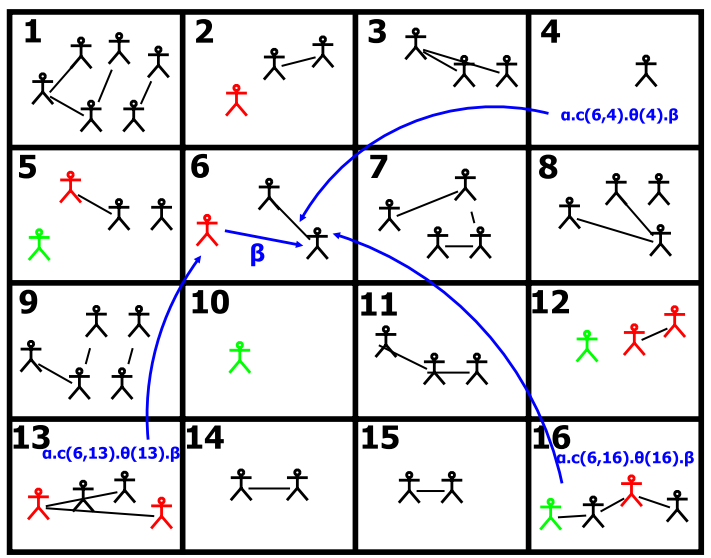

(b) Complex Networks Model

$I(4)$, respectively. Therefore, infection can transfer from region 4 to 6 via global transmission rate $\alpha c(6,4) I(4) \beta$. Similarly, $\alpha c(6,13) I(13) \beta$ and $\alpha c(6,16) I(16) \beta$ signifies the global transmission rate from region 13 and 16, respectively, to region 6 . On the other hand, b shows the complex network model: similar to fully mixed model, infection can transfer from region 4 to 6 via global transmission rate $\alpha c(6,4) \Theta(4) \beta$, where $\Theta$ takes care of the degree of the individual (see Sect. 3 for detail)

the mobility parameter (or direct connectivity) from one region to another. Along with presenting the mathematical proof of our model, we have performed extensive simulations on synthetic data. Moreover, we leverage our model to forecast the COVID-19 cases in Estonia ${ }^{1}$ and in the RhôneAlpes region $^{2}$ (one of the largest regions in France) by inferring the population mobility within the studied areas using mobile phone data. These various use cases demonstrate the model's ability to generalize on different types of data.

\subsection{Study highlights}

In our previous work Goel and Sharma 2020, we proposed fully mixed model that is composed of the (local) transmission rate of the infection $\beta$, and to cover the mobility aspect, we introduce parameters: (1) ' $\alpha$ ' which is a social connectivity parameter that signifies how well individuals are socially linked with each other, and (2) ' $c_{(i, j)}$ ' that represents individuals mobility from a region $j$ to another region $i$. Thus, the infection can transfer within the region with the transmission rate $\beta$ and can also be introduced from other regions through global transmission rate which depends upon $\alpha, c_{(i, j)}, I_{j}$ (fraction of infected at region $\mathrm{j}$ ) and $\beta$. In this study, we extend our work to complex networks model as well. With the help of Fig. 1, we illustrate our proposed models (fully mixed and

\footnotetext{
1 https://koroonakaart.ee/en.

${ }^{2}$ https://covidtracker.fr/dashboard-departements/?dep=71
} 
complex networks) for better understanding. We applied our model on a synthetic networks as well as on a new region, Rhône-Alpes in France considering the population density and the connectivity among different sectors, which is created using mobile phone data. Finally, we utilize our model to investigate the following questions:

- How social connectivity parameter ' $\alpha$ ' affects the fraction of individuals in different compartments (susceptible, infected and recovered) for fully mixed and complex networks model? We address this question by carefully examining the effect of $\alpha$ while keeping all the other parameters constant (Sects. 4.2 and 5.2).

- What are the outcomes of restricting mobility from the top-X percentile of strongly connected regions in fully mixed and complex networks model? We explore the outcomes of mobility restriction with the model and found that restricting the mobility of the top-10 percentile of strongly connected regions can reduce the number of infected individuals between 18 and 27\% (Sects. 4.2 and 5.2). Here, strongly connected regions are defined as the regions from which there is a higher number of regular commuters.

- What is the relationship between social connectivity parameter ' $\alpha$ ' and mobility restriction (of top-X percentile) from strongly connected regions? To address this question, we performed numerical simulation on the proposed mean-field equations for fully mixed model (Sect. 4.2, Fig. 4).

- How efficiently this model can perform in real scenar$i o s$ ? We answer this question by projecting the expected COVID-19 cases in Estonia and Rhône-Alpes region in France using the fully mixed model and compared the results with the real cases (Sects. 4.2.3 and 4.2.4).

The limitation of traditional compartmental epidemiological models is that they do not account for the role of reducing social connectivity (or isolation) and mobility restrictions during the outbreak of a pandemic such as COVID-19. The proposed model overcomes this constraint. We discovered that the reproduction number $R_{0}$ for a pandemic is influenced by social connectivity and mobility. We also discovered that restricting movement during a pandemic decreases the fraction of individuals in an infected compartment, and restricting social interaction (or isolation) slows the peak and reduces the number of infected individuals from the pandemic. We believe that these models can help to adopt a balanced strategy to address a pandemic crisis.

\subsection{Contributions}

The contribution of our work is twofold:
1. Previous mobility-based SIR model is proposed for fully mixed model (Goel and Sharma 2020). The main limitation of fully mixed model is that it assumes that every person at every location is linked to everyone else at that location. However, in reality, people interact with a limited number of people to form a complex network with non-trivial topological features that do not occur in simple networks such as lattices or random graphs but often occur in networks representing real systems (Albert and Barabási 2002). Therefore, this paper propose mobility-based SIR model for complex networks that is more realistic than fully mixed models.

2. Next, we show that our model perform well on real scenarios by projecting the expected COVID-19 cases at county scale (Estonia) and regional scale (Rhône-Alpes region in France).

\subsection{Paper organization}

The rest of the paper is organized as follows. Next, we discuss related works with respect to epidemic modeling. We then describe the model preliminaries and derivations in Sect. 3. Sections 4 and 5 present the evaluation results of our fully mixed and complex networks model, respectively. We conclude with a discussion of future directions in Sect. 6 .

\section{Related work}

In this section, we discuss relevant literature with respect to epidemic modeling which involves two different lines of work. The first involves agent-based modeling, while the second is related to compartmental based modeling. In the agent-based modeling, authors model epidemics by simulating the actions and interactions of autonomous agents (both individual or collective entities such as organizations or groups) with a view of assessing their effects on the system as a whole (Epstein 2009) by using transportation systems such as road networks (Eubank et al. 2004), airways (Chinazzi et al. 2020) etc. These models have been used for understanding various epidemics such as smallpox (Burke et al. 2006), influenza (Khalil et al. 2012), cholera (Crooks and Hailegiorgis 2014), and very recently COVID-19 (Chinazzi et al. 2020; Tuomisto et al. 2020).

In contrast to agent-based modeling, differential equation-based compartmental models have also been used for understanding epidemics, which is the basis of this work. This line of literature is mainly based on the classical SIR model proposed by Kermack and McKendrick (Kermack and McKendrick 1927) followed by Anderson and May (1979); Anderson et al. (1992). In Anderson and May (1979), the authors considered the host population as a dynamic variable rather than constant, as conventionally 
assumed, which provides a broader understanding of the population behavior during infectious disease. In their work in Anderson et al. (1992), authors discuss the idea of the basic reproductive rate, threshold about host densities, and modes of transmission.

Different variations of the SIR model have also been proposed to capture various real-world scenarios. For example, introducing a delay in the model to capture the incubation period during the spreading (Zhang et al. 2010; Xia et al. 2012; Liu 2015; Arquam et al. 2018) or the introduction of interventions such as antiviral drugs (Towers et al. 2011). In a different work to represent the nonlinear nature of epidemic spread, a SIR rumor spreading model was proposed in which tie strengths were dependent on nodes' degree (Singh and Singh 2012). Apart from SIRbased models, there exist several flavors of compartmental models, which represent different scenarios such as SIS (Nåsell 1996), where individuals do not recover and can become susceptible again. This model has also been studied using varying types of underlying topologies (Shi et al. 2008).

A set of works have also focused on exhibiting the epidemic spreading by using varying types of underlying network structures. For example, authors in Moreno et al. (2002); Barthélemy et al. (2005), and Vespignani (2012) used a scale-free network and in Li and Wang (2006) a small-world evolving networks for evaluating their epidemiological framework. In their work in Kiskowski (2014), researchers combine a discrete, stochastic SEIR (E stands for exposed) model with a three-scale community network model to demonstrate that the different regional trends may be explained by different community mixing rates. A detailed study concerning various epidemic models on varying topologies has been done in Pastor-Satorras et al. (2015).

In another line of work, the authors proposed models to understand epidemics based on the speed of growth. For example, in Viboud et al. (2016), authors applied their generalized-growth model to characterize the ascending phase of an outbreak on 20 different epidemics. Their findings revealed that sub-exponential growth is a common phenomenon, especially for pathogens that are not airborne. In another work Huang et al. 2016, researchers explained the rapid spread of H1N1 in 2009 around the world by using a flexible Bayesian, space-time, susceptible-infected-recovered (SIR) modeling approach. Gojovic et al. (2009) developed a simulation model of a pandemic (H1N1) 2009 outbreak in a structured population using demographic data from a medium-sized city in Ontario and epidemiologic influenza pandemic data. A set of work have also focused on mobility aspect during COVID-19 Goel and Sharma 2020; Arenas et al. 2020. In comparison to previous works, the proposed model introduces mobility and social connectivity parameters in both fully mixed and complex networks models, the key characteristics for turning epidemics into pandemics.

\section{Model preliminaries and derivations}

In this section, we first explain the classical SIR model and then discuss its limitations with respect to the absence of mobility and social connectivity parameters. Next, we describe our proposed model to understand the spreading of an infection during a pandemic.

In 1926, Kermack and McKendrick (1927) proposed the classical SIR model as follows:

$$
\frac{\mathrm{d} s(t)}{\mathrm{d} t}=-\beta s(t) i(t)
$$

$\frac{\mathrm{d} i(t)}{\mathrm{d} t}=\beta s(t) i(t)-\mu i(t)$

$\frac{\mathrm{d} r(t)}{\mathrm{d} t}=\mu i(t)$

where $s(t), i(t), r(t)$ are, respectively, the fraction of susceptible, infected and recovered population at time $t$. However, the classical SIR epidemic model proposed by Kermack and McKendrick (1927) does not consider the heterogeneity and topology of the real-world network. To overcome this limitation, we introduce the mobility and social connectivity parameters in our proposed model.

Let ' $l$ ' represent the total number of locations, and ' $c$ ' denote the connection (or individuals' mobility) between locations. The propagation of infection at each location is explained as follows: each healthy individual can get the infection either from an infected individual located in the same location (local transmission) or from an individual visiting from other connected locations (global transmission). The local transmission rate of infection is represented by $\beta$ and the recovery rate as $\mu$, with $\beta$ and $\mu \in$ $[0,1]$.

\subsection{Nonlinear dynamical system for fully mixed model}

Next, we discuss the local transmission of infection (Sect. 3.1.1), the global transmission (Sect. 3.1.2), and then the dynamical behavior of the nonlinear system of infection for fully mixed model (Sect. 3.1.3). 


\subsubsection{Local transmission}

Let $N_{i}$ be the population at location $i$, where $i \in l$, and the total population is divided into three compartments. The compartments for location $i$ at time $t$ are as follows:

1. $S_{i}(t)$ : the number of individuals susceptible or not yet infected. This compartment is referred as susceptible compartment.

2. $I_{i}(t)$ : the number of infected individuals which can further spread the disease to the individuals present in the susceptible compartment. This compartment is referred to as infected compartment.

3. $R_{i}(t)$ : the number of individuals who have been recovered from the infected compartment. This compartment is referred as recovered compartment.

Our assumptions regarding the transmission of an individual from one compartment to another compartment are as follows:

1. A healthy individual after becoming infected moves from susceptible to the infected compartment.

2. An individual can recover spontaneously at any time with recovery rate $\mu$. The recovery of an individual is independent of healthy and infected compartments' individuals.

3. Once the individual gets recovered, it will become immune to the disease and, thus, will not transmit the infection to individuals in the susceptible compartment.

4. In addition, this model ignores the demography that is birth or death of individuals. In other words, the population remains constant.

\subsubsection{Global transmission}

Let $j(j \subset l)$ represent a set of locations, which are connected to location $i$. Therefore, $\sum_{j} N_{j}$ is the maximum possible number of individuals connected to location $i$, from all the locations $j$. The parameter $c_{i, j}$ reflects the mobility of individuals from locations $j$ to location $i$. Global transmission depends upon this mobility parameter of individuals from one location to another. Similar to local transmission, $I_{j}$ is the number of individuals in the infected compartment in location $j$. Hence, total mobility of infected individuals from all the other connected locations to location $i$ is $\sum_{j} c_{i, j} \frac{I_{j}}{N_{j}}$.

Considering the above description, the chances of transmission of infection from all the connected locations to location $i$ are $\sum_{j} c_{i, j} \frac{I_{j}}{N_{j}} \beta$. This transmission further depends upon
Table 1 Parameters description for nonlinear dynamical system

\begin{tabular}{ll}
\hline Notations & Meaning \\
\hline$l$ & Number of locations \\
$c$ & Connection between locations \\
$S_{i}(t)$ & Number of susceptible individual at location $i$ at time $t$ \\
$I_{i}(t)$ & Number of infected individual at location $i$ at time $t$ \\
$R_{i}(t)$ & Number of recovered individual at location $i$ at time $t$ \\
$N_{i}(t)$ & Population at location $i$ at time $t$ \\
$\alpha$ & Social connectivity parameter \\
$\beta$ & Infection rate \\
$\mu$ & Recovery rate \\
$c_{i, j}$ & Individuals mobility from location $j$ to $i$ \\
\hline
\end{tabular}

the social connectivity $(\alpha)$ of all the individuals at location $i$. Therefore, the proportion of healthy individuals at location $i$ which can get infected from infected individuals from location $j$ is $\frac{\alpha \sum_{j} c_{i, j} \frac{I_{j}}{N_{j}} \beta}{N_{i}+\sum_{j} c_{i, j}}$. Thus, the mean-field equations for the dynamics of the pandemic, based on the above discussed interactions are the following:

$$
\begin{aligned}
\frac{\mathrm{d} S_{i}(t)}{\mathrm{d} t}= & -\frac{\beta S_{i}(t) I_{i}(t)}{N_{i}(t)}-\frac{\alpha S_{i}(t) \sum_{j} c_{i, j} \frac{I_{j}(t)}{N_{j}(t)} \beta}{N_{i}(t)+\sum_{j} c_{i, j}} \\
\frac{\mathrm{d} I_{i}(t)}{\mathrm{d} t}= & \frac{\beta S_{i}(t) I_{i}(t)}{N_{i}(t)}+\frac{\alpha S_{i}(t) \sum_{j} c_{i, j} I_{j}(t)}{N_{j}(t)} \beta \\
N_{i}(t)+\sum_{j} c_{i, j} & -\frac{\mu I_{i}(t)}{N_{i}(t)}
\end{aligned}
$$

$\frac{\mathrm{d} R_{i}(t)}{\mathrm{d} t}=\frac{\mu I_{i}(t)}{N_{i}(t)}$

where Eq. 4 describes the rate of change of susceptible individuals at location $i$, Eq. 5 refers to rate of change of infected individuals, and Eq. 6 explains the rate of change of recovered individuals at location $i$. The reader can refer to Table 1 for notations and their meaning.

\subsubsection{Dynamical behavior of the linear system}

Equations (4-6) represent a nonlinear dynamical system of pandemic spreading, where, at any time $t$,

$S_{i}(t)+I_{i}(t)+R_{i}(t)=N_{i}(t)$

In order to solve mean-field Eqs. (4-6), following assumptions are made (please note that these assumptions are not considered during our experiments): 
1. Initially, the population at all locations is equal to $N(t)$ at time $t$.

2. Individuals in infected compartments are equal to $I(t)$ at all locations at time $t$ and $\sum_{j} I_{j}=|j| \cdot I_{j}=k I_{j}$, where $k$ is the number of locations connected to location $i$, that is, $k=|j|$.

3. The mobility of individuals from one location to another location is a fraction of total population $N$. Let $n$ be the sum of fraction of population mobility from $|k|$ locations. Then, the total individuals mobility from set of locations $j$ to $i$ is $n * N$. Therefore, $\sum_{j} c_{i, j}=n N$.

By considering the above assumptions, Eqs. 4 and 6 can be written as

$\frac{\mathrm{d} S_{i}(t)}{\mathrm{d} t}=-\frac{\beta S_{i}(t) I(t)}{N(t)}-\frac{\alpha S_{i}(t) n N(t) k \frac{I(t)}{N(t)} \beta}{N(t)+n N(t)}$

$\frac{\mathrm{d} R_{i}(t)}{\mathrm{d} t}=\frac{\mu I(t)}{N(t)}$

From Eqs. 8 and 9

$\frac{\mathrm{d} S_{i}(t)}{\mathrm{d} R_{i}(t)}=-\frac{\beta S_{i}(t)}{\mu}-\frac{\alpha S_{i}(t) n k \beta}{\mu(1+n)}$

$=-\frac{\beta S_{i}(t)}{\mu}\left[1+\frac{\alpha n k}{1+n}\right]$

$=-\frac{\beta S_{i}(t)}{\mu}\left[\frac{1+(1+\alpha k) n}{1+n}\right]$

For simplicity, Eq. 12 can be written as:

$\frac{\mathrm{d} S(t)}{\mathrm{d} R(t)}=-\frac{\beta S(t)}{\mu}\left[\frac{1+(1+\alpha k) n}{1+n}\right]$

Equation 13 can be rewritten as

$S=S_{0} e^{-\frac{\beta}{\mu} R\left[\frac{1+(1+\alpha k) n}{1+n}\right]}$

$\frac{\mathrm{d} R}{\mathrm{~d} t}=\mu\left(N-R-S_{0} e^{-\frac{\beta}{\mu} R\left[\frac{1+(1+\alpha k) n}{1+n}\right]}\right)$

Solving Eq. 15, we get

$t=\frac{1}{\mu} \int_{0}^{R} \frac{\mathrm{d} R}{N-R-S_{0} e^{-\frac{\beta}{\mu} R\left[\frac{1+(1+\alpha k) n}{1+n}\right]}}$

As pandemic arrives at steady state when $t \longrightarrow \infty$ hence $\frac{d R}{d t}$ $=0$ and $R_{\infty}=C$, where $C$ is a constant:
$N-R_{\infty}=S_{0} e^{-\frac{\beta}{\mu} R_{\infty}\left[\frac{1+(1+\alpha k) n}{1+n}\right]}$

Let the initial conditions be: $R(0)=0, I(0)=I$ and $S(0)=N-I \approx N$. Therefore, Eq. 17 can be written as:

$R_{\infty}=N-N e^{-\frac{\beta}{\mu} R_{\infty}\left[\frac{1+(1+\alpha k) n}{1+n}\right]}$

Normalizing Eq. 18 by dividing by total population $N$ gives:

$r_{\infty}=1-1 e^{-R_{0} r_{\infty}}$

Therefore, the reproduction number $R_{0}$ is

$R_{0}=\frac{\beta}{\mu}\left[\frac{1+(1+\alpha k) n}{1+n}\right]$

In case there is no social connectivity to other locations ( $\alpha=0$ or $k=0$ or $n=0$ ), then the mobility SIR model will become the standard SIR model and the reproduction number is $R_{0}=\frac{\beta}{\mu}$. Therefore, the reproduction number is directly proportional to social connectivity parameter $\alpha$, number of connected locations $k$, and depends upon individuals' mobility during a pandemic.

The fully mixed model that we presented in this section has one key limitation, i.e., it assumes that every person at every location is linked to everyone else at that location. However, in reality, people interact with a limited number of people to form a complex network with non-trivial topological features that do not occur in simple networks such as lattices or random graphs but often occur in networks representing real systems Albert and Barabási 2002. Therefore, in the next section, we propose mobility-based SIR model for complex networks.

\subsection{Nonlinear dynamical system for complex networks}

In this section, we discuss the local transmission of infection (Sect. 3.2.1), the global transmission (Sect. 3.2.2), and then the dynamical behavior of the nonlinear system of infection by considering complex networks interactions at each location (Sect. 3.2.3).

\subsubsection{Local transmission}

Let $N_{i}$ be the population at location $i$, where $i \in l$, and $k$ is the degree of each individual, where $k \in \mathbb{W}$ (whole numbers). The total population is divided into three compartments. The compartments for location $i$ at time $t$ are as follows: 
Table 2 Parameters description for nonlinear dynamical system for complex network

\begin{tabular}{ll}
\hline Notations & Meaning \\
\hline$l$ & Number of locations \\
$c$ & Connection between locations \\
$S_{i}(k, t)$ & Number of susceptible individual of degree $k$ at location $i$ at time $t$ \\
$I_{i}(k, t)$ & Number of infected individual of degree $k$ at location $i$ at time $t$ \\
$R_{i}(k, t)$ & Number of recovered individual of degree $k$ at location $i$ at time $t$ \\
$N_{i}(k, t)$ & Population of degree $k$ at location $i$ at time $t$ \\
$\alpha$ & Social connectivity parameter \\
$\beta$ & Infection rate \\
$\mu$ & Recovery rate \\
$c_{i, j, k}$ & Mobility of individuals of degree $k$ from location $j$ to $i$ \\
$\Psi\left(k^{\prime}\right)$ & Infectivity of a node with degree $k^{\prime}$ \\
\hline
\end{tabular}

1. $S_{i}(k, t)$ : the number of individuals susceptible or not yet infected at time $t$ having degree $k$. This compartment is referred as susceptible compartment.

2. $I_{i}(k, t)$ : the number of infected individuals at time $t$ having degree $k$, which can further spread the disease to the individuals present in the susceptible compartment. This compartment is referred to as infected compartment.

3. $R_{i}(k, t)$ : the number of individuals at time $t$ having degree $k$, who have been recovered from infected compartment. This compartment is referred as recovered compartment.

Our assumptions regarding the transmission of an individual from one compartment to another compartment are same as discussed in Sect. 3.1.1.

\subsubsection{Global transmission}

Let $j(j \subset l)$ represent a set of locations, which are connected to location $i$. Therefore, $\sum_{j} N_{j}(k)$ is the maximum possible number of individuals of degree $k$ connected to location $i$, from all the locations $j$. The parameter $c_{i, j, k}$ reflects the mobility of individuals of degree $k$ from locations $j$ to location $i$. Global transmission depends upon this mobility parameter of individuals from one location to another. Similar to local transmission, $I_{j}$ is the number of individuals in the infected compartment in all the locations $j$. Hence, total mobility of infected individuals of degree $k$ from all the other connected locations to location $i$ is $\sum_{j} c_{i, j, k} \frac{I_{j}(k)}{N_{j}(k)}$.

Considering the above description, the chances of transmission of infection from all the connected locations to location $i$ are $\sum_{j} c_{i, j, k} \frac{I_{j}(k)}{N_{j}(k)} \beta$. This transmission further depends upon the social connectivity $(\alpha)$ of all the individuals at location $i$. Therefore, the proportion of healthy individuals at location $i$ which can get infected from infected individuals from location $j$ is $\frac{\alpha \sum_{j} c_{i, j, k} \sum_{j}(k)}{N_{i}(k)+\sum_{j} c_{i, j, k}} \beta$. Thus, the mean-field equations for the nonlin- ear dynamics of the pandemic, based on the above discussed interactions are the following:

$$
\begin{aligned}
\frac{d S_{i}(k, t)}{d t}= & -\frac{\beta S_{i}(k, t) \Theta_{i}(t)}{N_{i}(k, t)} \\
& -\frac{\alpha S_{i}(k, t) \sum_{j} c_{i, j, k} \frac{\Theta_{j}(t)}{N_{j}(k, t)} \beta}{N_{i}(k, t)+\sum_{j} c_{i, j, k}} \\
\frac{d I_{i}(k, t)}{d t}= & \frac{\beta S_{i}(k, t) \Theta_{i}(t)}{N_{i}(k, t)} \\
& +\frac{\alpha S_{i}(k, t) \sum_{j} c_{i, j, k} \frac{\Theta_{j}(t)}{N_{j}(k, t)} \beta}{N_{i}(k, t)+\sum_{j} c_{i, j, k}} \\
& -\frac{\mu I_{i}(k, t)}{N_{i}(k, t)}
\end{aligned}
$$

$\frac{d R_{i}(k, t)}{d t}=\frac{\mu I_{i}(k, t)}{N_{i}(k, t)}$

where

$\Theta_{i}(t)=\sum_{k^{\prime}=1}^{k} \frac{\Psi\left(k^{\prime}\right) P\left(\frac{k^{\prime}}{k}\right) I_{i}\left(k^{\prime}, t\right)}{k^{\prime}}$

$P\left(\frac{k^{\prime}}{k}\right)=\frac{k^{\prime} P\left(k^{\prime}\right)}{<k>}$

where Eq. 21 describes the rate of change of susceptible individuals of degree $k$ at location $i$, and Eq. 22 refers to rate of change of infected individuals of degree $k$, and Eq. 23 explains the rate of change of recovered individuals of degree $k$ at location $i$. Please refer Table 2 for notations and their meaning. 


\subsubsection{Dynamical behavior of the nonlinear system for complex networks}

Equations (21-23) represents nonlinear dynamical system of pandemic spreading for complex networks, where, at any time $t$ :

$S_{i}(t)+I_{i}(t)+R_{i}(t)=N_{i}(t)$

where

$X(t)=\sum_{k} X(k, t) ; X \in\{S, I, R, N\}$

In order to solve mean-field Eqs. (21-23) similar assumptions as in Sect. 3.1.3 are made. By considering such assumptions, Eqs. 21, 22 and 23 can be written as

$$
\begin{aligned}
\frac{\mathrm{d} S_{i}(k, t)}{\mathrm{d} t}= & -\frac{\beta S_{i}(k, t) \Theta(t)}{N(k, t)} \\
& -\frac{\alpha S_{i}(k, t) n N(k, t) m \frac{\Theta(t)}{N(k, t)} \beta}{N(k, t)+n N(k, t)}
\end{aligned}
$$

$$
\begin{aligned}
\frac{\mathrm{d} I_{i}(k, t)}{\mathrm{d} t}= & \frac{\beta S_{i}(k, t) \Theta(t)}{N(k, t)} \\
& +\frac{\alpha S_{i}(k, t) n N(k, t) m \frac{\Theta(t)}{N(k, t)} \beta}{N(k, t)+n N(k, t)} \\
& -\frac{\mu I(k, t)}{N(k, t)}
\end{aligned}
$$

$$
\frac{\mathrm{d} R_{i}(k, t)}{\mathrm{d} t}=\frac{\mu I(k, t)}{N(k, t)}
$$

From Eqs. 28 and 30

$$
\begin{aligned}
& \frac{\mathrm{d} S_{i}(k, t)}{\mathrm{d} R_{i}(k, t)}=-\frac{\beta S_{i}(k, t) \Theta(t)}{\mu I(k, t)}-\frac{\alpha S_{i}(k, t) n m \Theta(t) \beta}{\mu(1+n) I(k, t)} \\
& =-\frac{\beta S_{i}(k, t) \Theta(t)}{\mu I(k, t)}\left[1+\frac{\alpha n m}{1+n}\right] \\
& =-\frac{\beta S_{i}(k, t) \Theta(t)}{\mu I(k, t)}\left[\frac{1+(1+\alpha m) n}{1+n}\right]
\end{aligned}
$$

For simplicity, Eq. 33 can be written as:

$$
\frac{\mathrm{d} S(k, t)}{\mathrm{d} R(k, t)}=-\frac{\beta S(k, t) \frac{\left\langle k^{2}\right\rangle}{\langle k\rangle} I(k, t)}{\mu I(k, t)}\left[\frac{1+(1+\alpha m) n}{1+n}\right]
$$

$\frac{\mathrm{d} S(k, t)}{\mathrm{d} R(k, t)}=-\frac{\beta S(k, t) \frac{\left\langle k^{2}\right\rangle}{\langle k\rangle}}{\mu}\left[\frac{1+(1+\alpha m) n}{1+n}\right]$

Equation 35 can be rewritten as

$S=S_{0} e^{-\frac{\beta \frac{\left\langle k^{2}\right\rangle}{k \mid}}{\mu} R\left[\frac{1+(1+a m) n}{1+n}\right]}$

$\frac{\mathrm{d} R}{\mathrm{~d} t}=\mu\left(N-R-S_{0} e^{-\frac{\beta \frac{\left\langle k^{2}\right\rangle}{k\rangle}}{\mu} R\left[\frac{1+(1+\alpha m) n}{1+n}\right]}\right)$

Solving Eq. 37, we get

$t=\frac{1}{\mu} \int_{0}^{R} \frac{\mathrm{d} R}{N-R-S_{0} e^{-\frac{\beta \frac{\left\langle k^{2}\right\rangle}{k k}}{\mu} R\left[\frac{1+(1+a m) n}{1+n}\right]}}$

As pandemic arrives at steady state, when $t \longrightarrow \infty$, hence $\frac{\mathrm{d} R}{\mathrm{~d} t}$ $=0$ and $R_{\infty}=C$, where $C$ is a constant.

$N-R_{\infty}=S_{0} e^{-\frac{\beta \frac{\left\langle k^{2}\right\rangle}{k k\rangle}}{\mu} R_{\infty}\left[\frac{1+(1+\alpha m) n}{1+n}\right]}$

Let initial conditions are $R(0)=0, I(0)=I$ and $S(0)=N-I \approx N$. Therefore, Eq. 39 can be written as

$R_{\infty}=N-N e^{-\frac{\beta \frac{\left\langle k^{2}\right\rangle}{\langle k\rangle}}{\mu} R_{\infty}\left[\frac{1+(1+\alpha m) n}{1+n}\right]}$

Normalizing Eq. 40 by dividing by total population $N$ gives:

$r_{\infty}=1-1 e^{-R_{0} r_{\infty}}$

Therefore, the reproduction number $R_{0}$ is

$R_{0}=\frac{\beta \frac{\left\langle k^{2}\right\rangle}{\langle k\rangle}}{\mu}\left[\frac{1+(1+\alpha m) n}{1+n}\right]$

The $R_{0}$ is called basic reproduction number which determines the spread of infection. When $R_{0}>1$, the propagation occurs at a fast rate. When $R_{0}=1$, the propagation happens at a slow rate. When $R_{0}<1$, the propagation finishes. In case there is no social connectivity to other locations ( $\alpha=0$ or $m=0$ or $n=0$ ), then the mobility SIR model for complex networks gives the reproduction number as $R_{0}=\frac{\beta \frac{\left\langle k^{2}\right\rangle}{\langle k\rangle}}{\mu}$. Therefore, the basic reproduction number is directly proportional to social connectivity parameter $\alpha$, number of connected locations $m$, depends upon individuals' mobility during a pandemic, and degree of an individual in a complex network. 


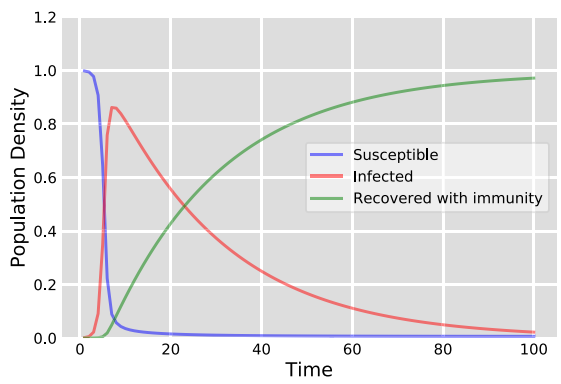

(a) $\alpha=1$

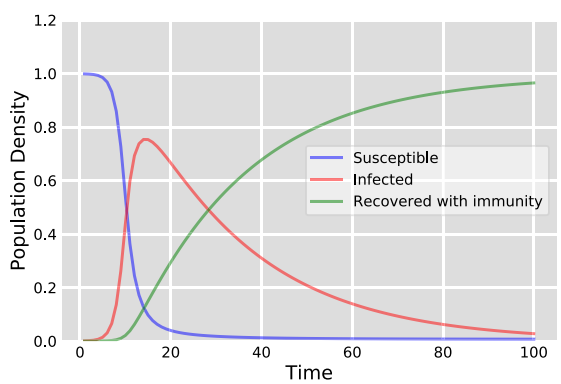

(d) $\alpha=0.4$

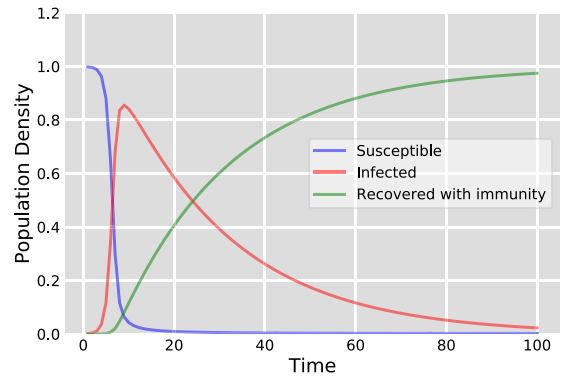

(b) $\alpha=0.8$

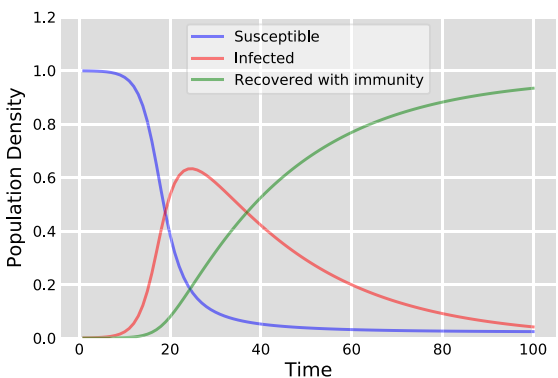

(e) $\alpha=0.2$

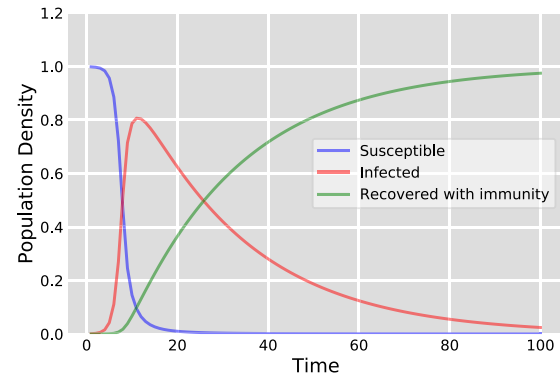

(c) $\alpha=0.6$

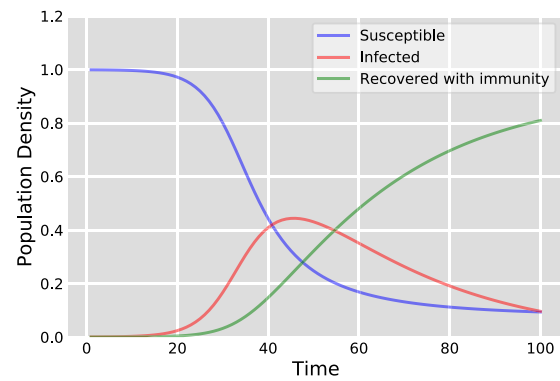

(f) $\alpha=0.1$

Fig. 2 Pandemic origin from random location: effect of Social connectivity parameter ' $\alpha$ '

\section{Evaluation of fully mixed model}

In this section, we first explain our experimental setup, and next, we discuss the results of our simulation conducted using the proposed model for non-complex network on synthetic data. In addition, we also show results of our model when applied for predicting the number of COVID-19 cases at country level (Estonia) and regional level (Rhône-Alpes region in France).

\subsection{Experimental setup}

For the analysis, we created an aggregated flow matrix whose cells represent the number of trips of individuals per day from origins to destinations. We call this matrix Origin-Destination (OD) matrix. The synthetic OD matrix considered for our experiment follows a random distribution. Furthermore, three different techniques are considered for selecting the seed infection location:

1. Pandemics origin from a random location: In this, a random location is selected as seed infection location, and a small fraction of individuals were infected at that location.

2. Pandemics origin from a weakly connected location: Here, the seed location is selected strategically, i.e., in a location which is weakly connected to other locations. This implies the least mobility of individuals from this location to other locations.

3. Pandemics origin from a strongly connected location: In this also, the seed location is selected strategically, i.e., in a location which is strongly connected to other locations. This signifies that the highest mobility of individuals from a location is considered for the selection of the infection seed.

Our simulation is oriented toward addressing the following questions:

- How social connectivity parameter ' $\alpha$ ' affects the fraction of individuals in different compartments (susceptible, infected and recovered) during a pandemic?

- What are the outcomes of restricting the mobility (for top-X percentile) of strongly connected locations?

- What is the relationship between social connectivity parameter ' $\alpha$ ' and the mobility restriction (top-X percentile of strongly connected locations)?

- How efficiently this model can perform in real scenarios? We answer this question by projecting the expected COVID-19 cases for Estonia and Rhône-Alpes region in France. 


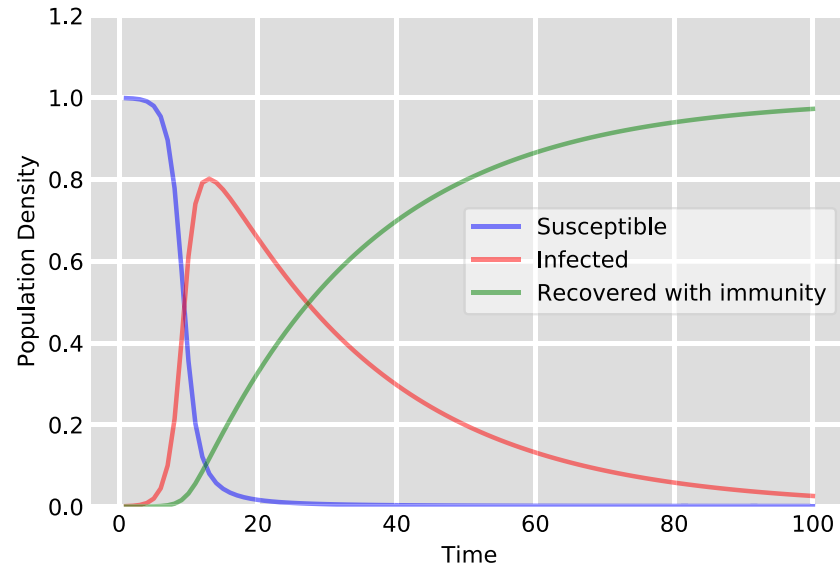

(a) $0 \%$ Locations Quarantine

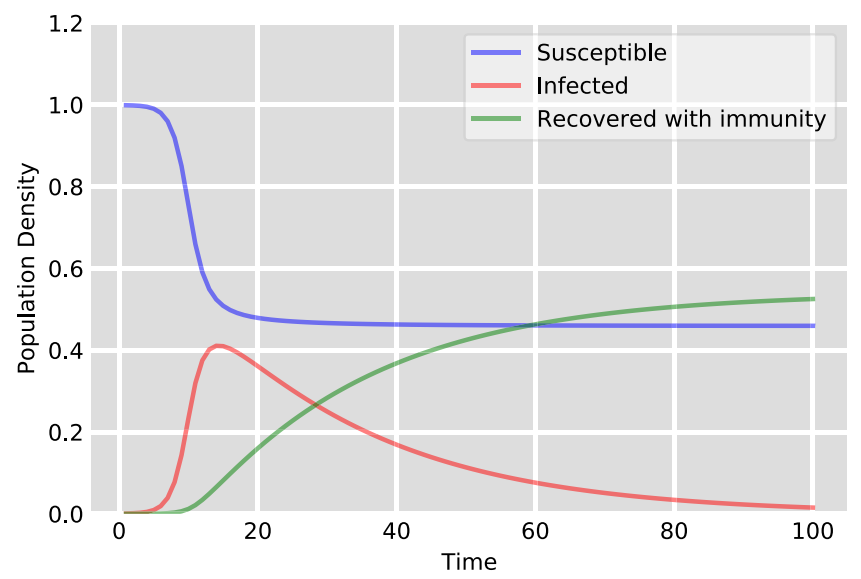

(c) $20 \%$ Locations Quarantine

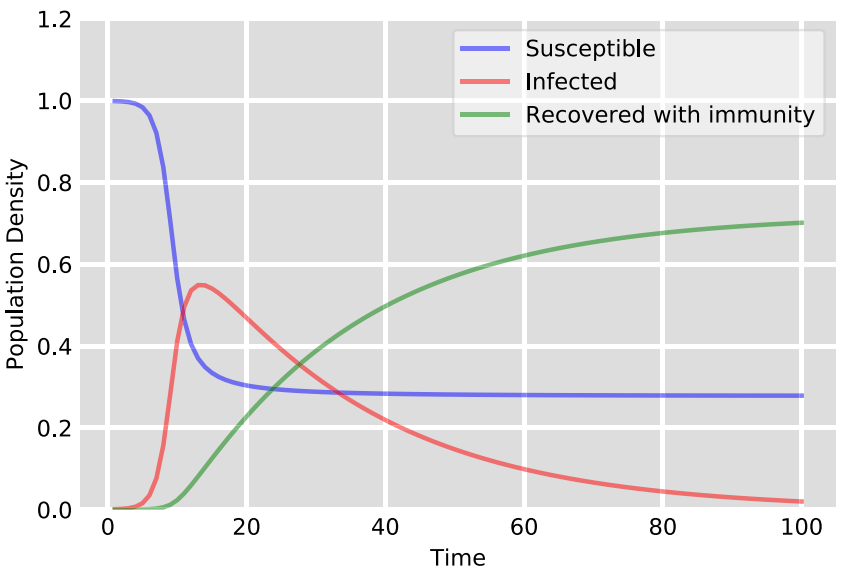

(b) $10 \%$ Locations Quarantine

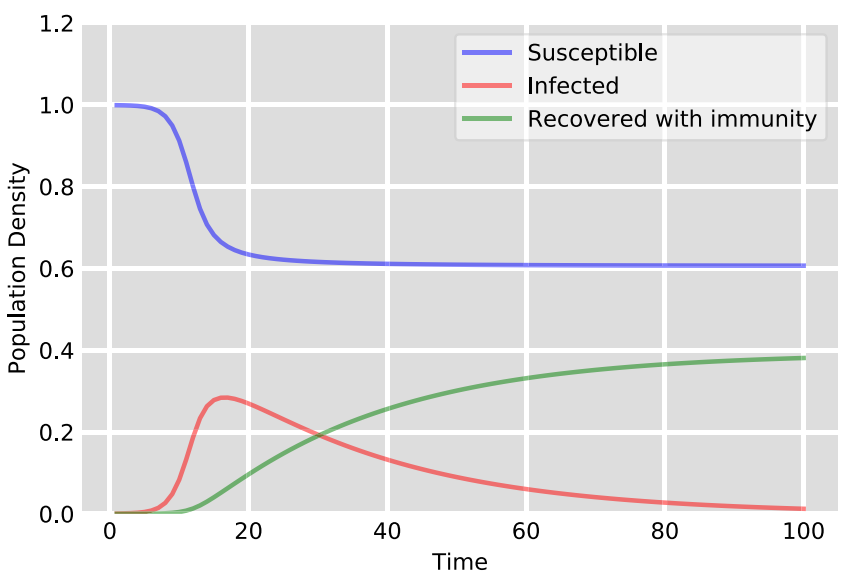

(d) $30 \%$ Locations Quarantine

Fig. 3 Pandemic origin from random location: effect of quarantine strongly connected locations

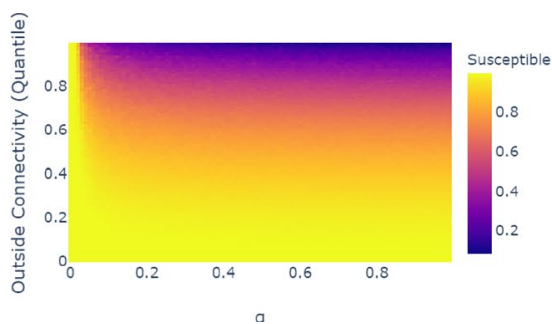

(a) Susceptible Compartment

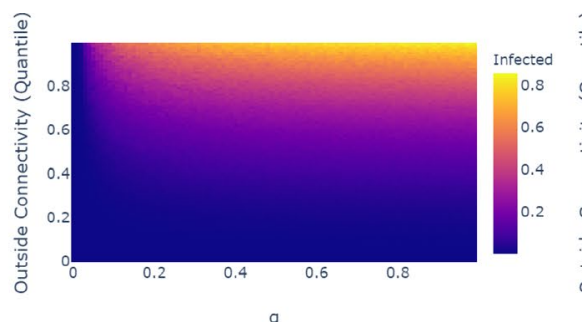

(b) Infected Compartment

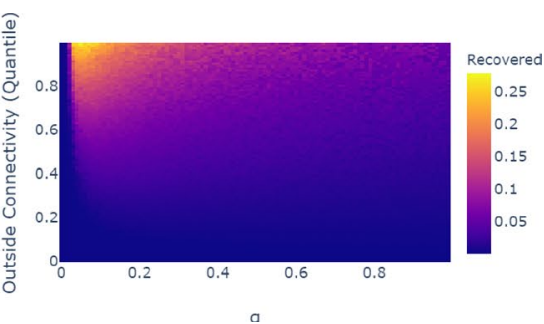

(c) Recovered Compartment

Fig. 4 Pandemic origin from random location: numerical simulation of relationship between $\alpha$ and quarantine

\subsection{Results}

We perform various simulation experiments to explain the proposed model on $O D$ matrix by using previously discussed techniques for selecting the seed infection location. It is to be noted that the model will behave as a standard SIR model in two cases, (i) if $\alpha=0$, (ii) if the mobility is reduced to 100 percentile (that is no mobility allowed) from connected locations. 


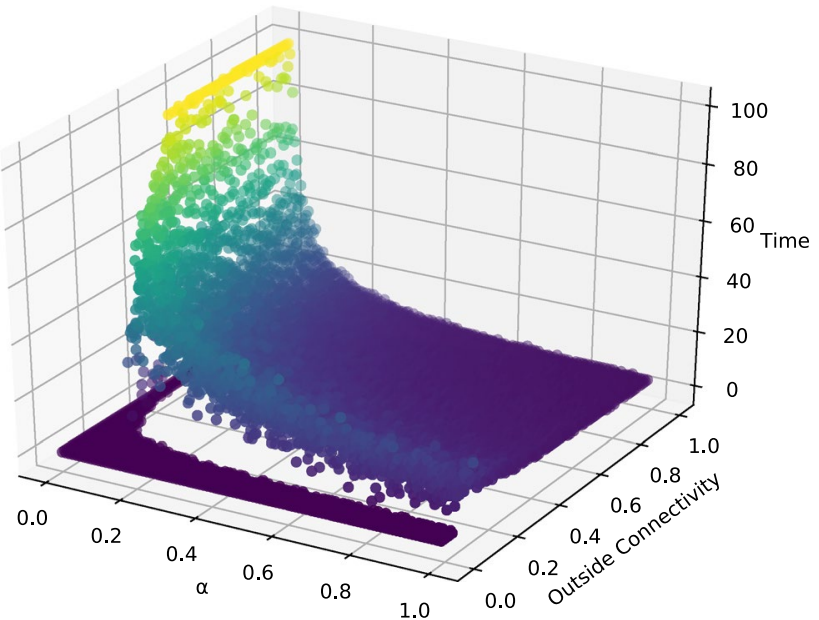

Fig. 5 For different combinations of $\alpha$ and quarantine percentile, number of days required to reach peak of infected compartment

\subsubsection{Pandemic origins from random location}

Figure 2 displays the influence of the social connectivity parameter ' $\alpha$ ' while keeping the other parameters constant. Figure $2 \mathrm{a}$, $\mathrm{f}$ shows the pandemic dynamics with different values of $\alpha$ starting with $\alpha=1$ to $\alpha=0.1$. We observe that the peak of the infected compartment decreases significantly, as the $\alpha$ decreases, and it also takes longer to reach its peak. This indicates that there is a positive impact of lockdown in controlling a pandemic.
The effect of restricting the mobility from the top-X percentile of highly connected locations with other locations is shown in Fig. 3. Figure 3a, d displays the pandemic dynamics with different percentile of mobility restrictions of highly connected locations starting with 0-30\% (keeping $\alpha=0.5$ ). We observe that in the case of a pandemic, restricting the mobility from the top-10 percentile of highly connected locations can reduce the number of individuals who can get infected to $27 \%$. Therefore, quarantine plays a vital role during pandemics.

In order to understand the relationship between $\alpha$ and mobility restriction from strongly connected locations, we performed the numerical simulation of the proposed mean-field equations (see Fig. 4). It shows the fraction of population in various compartments at time $t \rightarrow \infty$ for various value combinations of $\alpha$ and mobility restriction. From Fig. 4, we can infer that the social connectivity parameter ' $\alpha$ ' and mobility plays both a fundamental role in determining the dynamics of the pandemics. Therefore, it is advisable to follow a dual strategy approach during a pandemic outbreak as controlling mobility reduces the fraction of infected individuals, and $\alpha$ delays the peak. Furthermore, we analyzed the number of days required to reach the point where the highest fraction of individuals get infected (see Fig. 5). This indicates that mobility restrictions and minimal social contact will postpone the pandemic's peak and will give sufficient time for preparations, especially for the health sector.

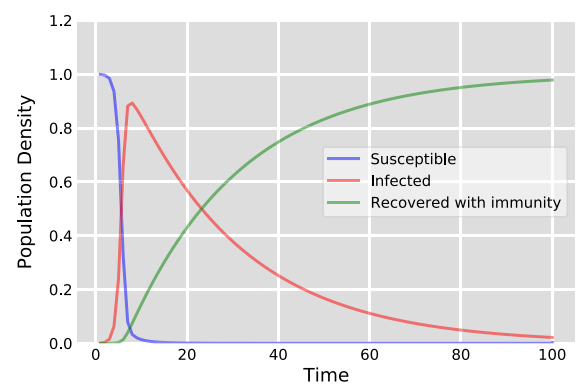

(a) $\alpha=1$

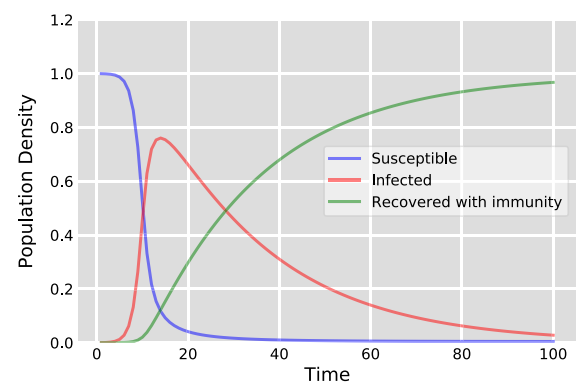

(d) $\alpha=0.4$

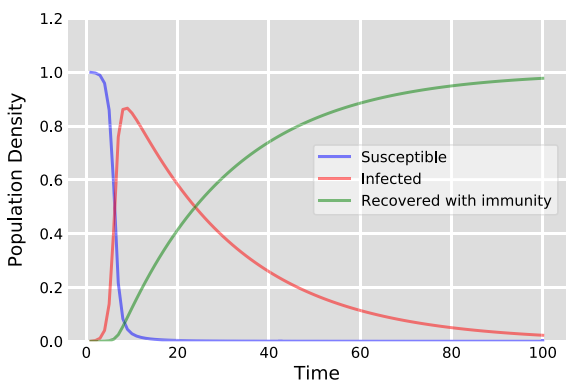

(b) $\alpha=0.8$

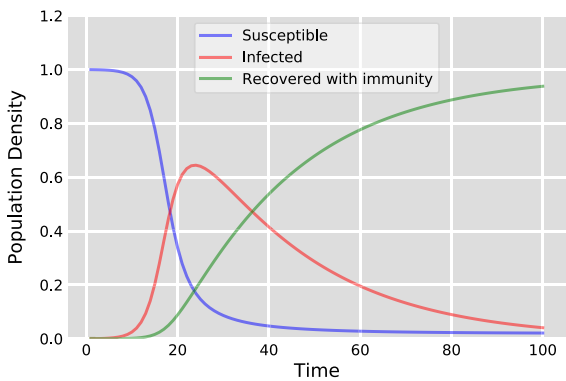

(e) $\alpha=0.2$

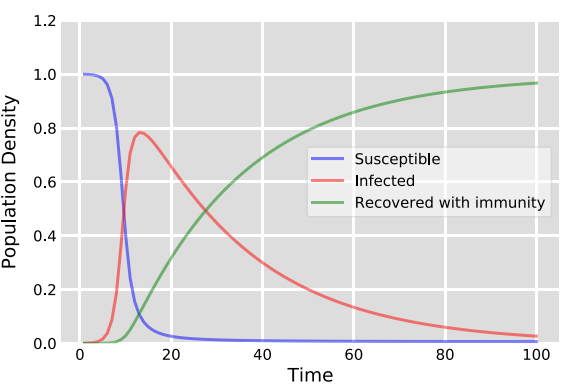

(c) $\alpha=0.5$

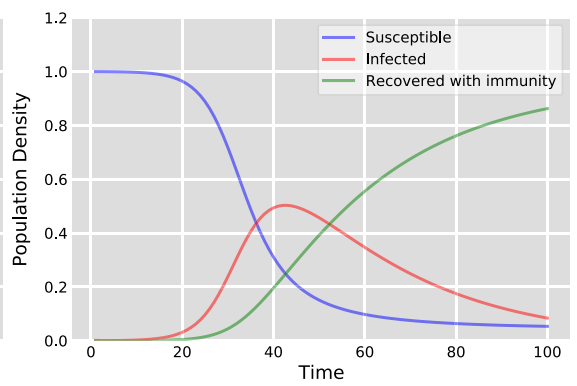

(f) $\alpha=0.1$

Fig. 6 Pandemic origin from weakly connected location: effect of social connectivity parameter ' $\alpha$ ' 


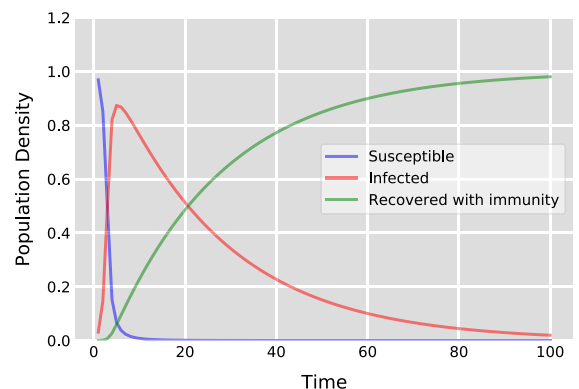

(a) $\alpha=1$

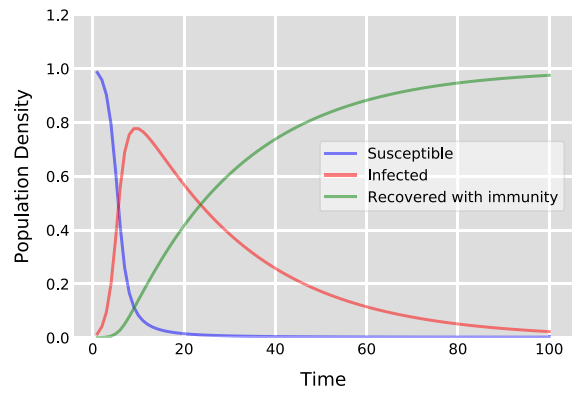

(d) $\alpha=0.4$

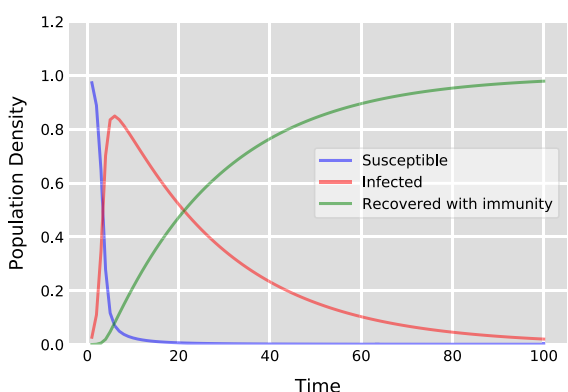

(b) $\alpha=0.8$

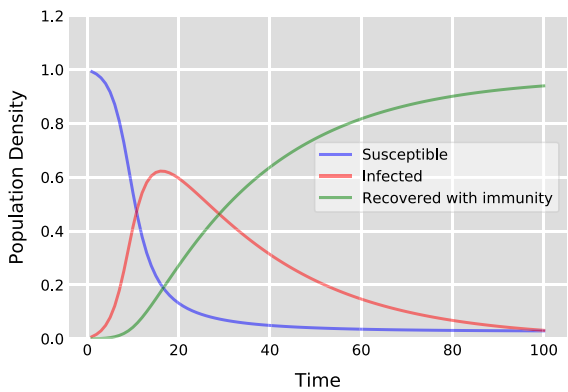

(e) $\alpha=0.2$

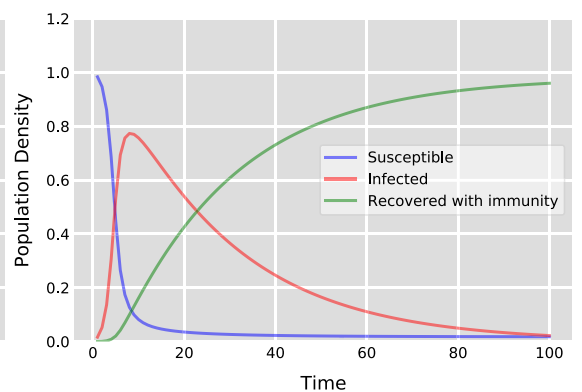

(c) $\alpha=0.5$

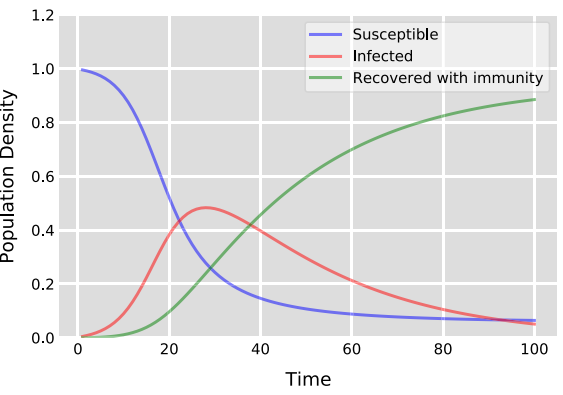

(f) $\alpha=0.1$

Fig. 7 Pandemic origin from strongly connected location: effect of social connectivity parameter ' $\alpha$ '

\subsubsection{Pandemic origins from weakly and strongly connected locations}

Figures 6, and 7 display the influence of the social communication parameter ' $\alpha$ ' while keeping the other parameters constant for weakly and strongly connected locations, respectively. Figure 6a, f shows the pandemic dynamics with different values of $\alpha$ starting with $\alpha=1$ to $\alpha=0.1$ for weakly connected locations. Similarly, Fig. 7a, f shows the pandemic dynamics with different values of $\alpha$ starting with $\alpha=1$ to $\alpha=0.1$ for strongly connected locations.

It can be noted that when a pandemic originates from a weakly connected location, it takes longer to reach its peak compared to when it starts from a strongly connected location. This shows that the location of origin also plays an important role during a pandemic. Similar to a random location, reducing mobility from the highly connected locations by 10 percentile can reduce the number

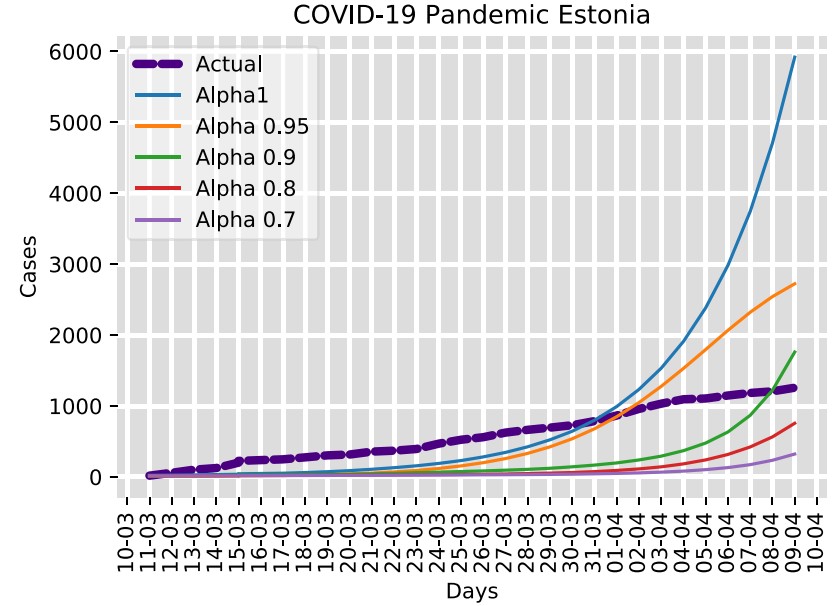

Fig. 8 COVID-19 cases in Estonia

of infected individuals between 18 and $27 \%$ for weakly and strongly connected locations, respectively. 


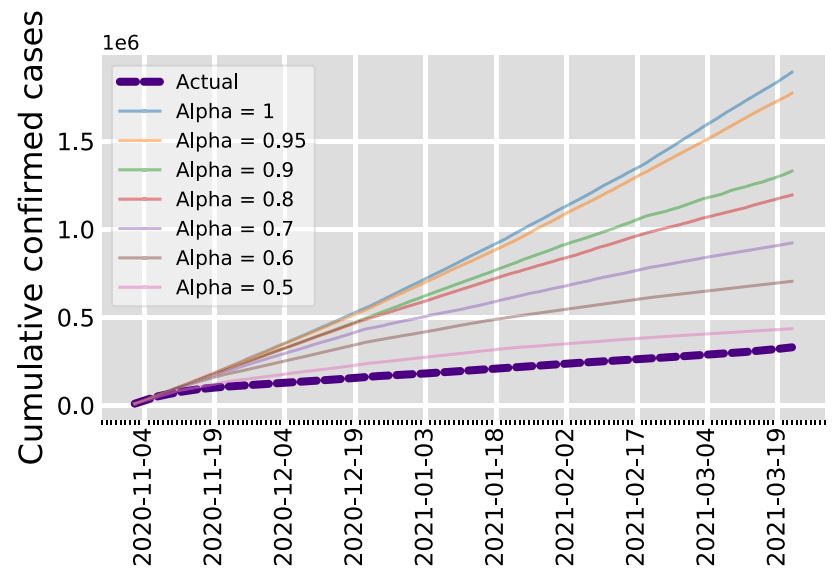

Fig. 9 COVID-19 cases in Rhône-Alpes region In France

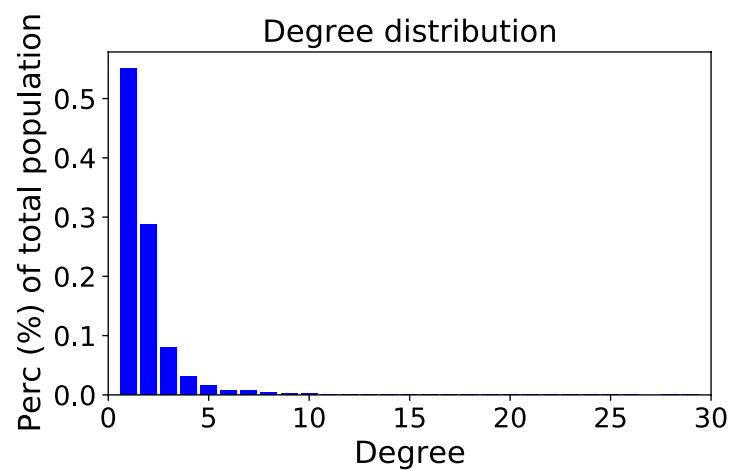

(a) Degree distribution of nodes.
For simulation, we created the $O D$ matrix between counties of Estonia using call data records Goel et al. 2021; Hiir et al. 2019. Furthermore, these call interactions are converted into population mobility between counties using Estonian population data from census Estonia 2018. For the local transmission of the virus (within the county), we consider the reproduction number $R_{0}=2.5$ (Organization W.H.et al. 2020).

Cases reported until March 11, 2020 are considered as an initial condition for the model. The reason behind selecting March 11, 2020 as initial condition is that, till this date no local transmission of the virus was reported ${ }^{3}$. Till the day of initial condition, the Estonian Health Board confirmed 13 cases in Harju and two cases in Tartumaa and Saaremaa each ${ }^{4}$. During the simulation, the number of cases in all other counties are initialized to zero. The infection rate $\beta$ and recovery rate $\mu$

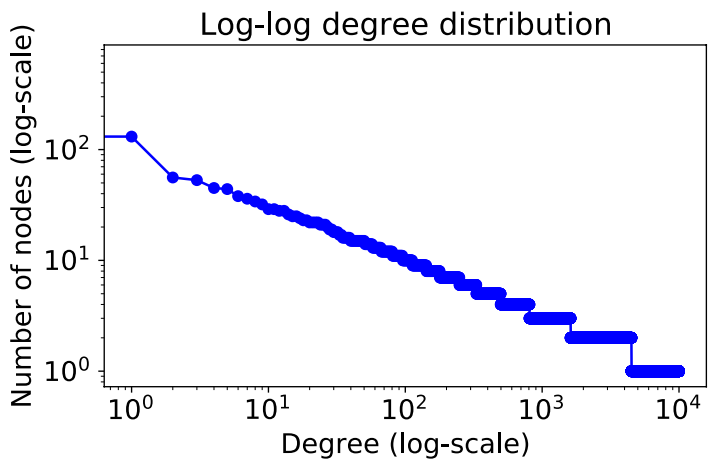

(b) Log-log degree distribution.

Fig. 10 The degree distribution of our synthetically generated network shows that it follows power law distribution

\subsubsection{Case study of Estonia}

To demonstrate the usability of the model, we applied it to real-time data of Estonia's COVID-19 cases. Figure 8 shows the actual number of cases and the cases forecast by the model using different values for $\alpha$ and mobility percentile. For example, $\alpha=0.95$ indicates that the social connectivity of individuals is reduced by $5 \%$ and also top- 5 percentile of strongly connected locations are restricted from mobility. Similarly, $\alpha=0.7$ implies that the social connectivity of individuals is reduced by $30 \%$, and also the top-30 percentile of strongly connected locations have introduced restricted mobility. are adjusted according to the value of $R_{0}$ for COVID-19. The reported cases in Estonia as well as the forecast cases using the model are shown in Fig. 8 until April 9, 2020. It can be noticed that the model predicted much higher cases of COVID-19 if no restrictions are introduced $(\alpha=1)$. However, as the restrictions were introduced by the Government, ${ }^{5}$ the number of cases got damped (Actual). Thus, the applicability of this model is to forecast a range of predicted number of cases which can help the government and health agencies to understand the impact and introduce proportional interventions to restrict the spread of the epidemic.

\footnotetext{
${ }_{3}$ https://www.err.ee/1063204/terviseamet-eestis-on-kinnitatud-27koroonajuhtu-ja-kohalik-levik.

${ }^{4}$ https://www.terviseamet.ee/et/uuskoroonaviirus.

${ }^{5}$ https://www.valitsus.ee/en/news
} 


\subsubsection{Case study of Rhône-Alpes region in France}

We also applied our model to real-time data of Rhône-Alpes region's COVID-19 cases. Figure 9 shows the actual number of cases and the cases forecast by the model using different values for $\alpha$ and mobility percentile. The region is divided into 14 sectors. For simulation purpose, we again considered the $O D$ matrix between the sectors of the region obtained via network signaling data of Orange (the largest telecommunications provider in France) and census data. ${ }^{6}$ This OD matrix has been built using the approach proposed by Fekih et al. in Fekih et al. (2020). The latter uses a heuristic-based approach to extract trips between sectors at individual level from mobile phone passive traces. As the signaling data collected by the telecommuting operator covers around 30\% of the whole population of the region, the resulting trips have been re-scaled using the census data. Finally, after an aggregation step, we obtain the regional OD matrix used in this study.

For privacy matters, the number of COVID-19 cases is not reported in France at a fine spatiotemporal resolution in publicly available data. ${ }^{7}$ Instead, the dataset only reports cumulative values of COVID-19 cases on a 7-day rolling window for each area of the administrative segmentation of the French territory. In addition, the number of cases is reported discretely, i.e., as a range of values between a lower and upper bound containing the real value. Therefore, in order to obtain a daily estimation of the number of COVID-19 cases per each sector of the analyzed RhoneAlpes region, two main assumptions have been made. On the one hand, we consider the number of cases as the mid value between the lower and upper bound of the reported range for the given area on a specific day. On the other hand, we replaced the 7-day rolling time window by the median day (i.e., the 4 th day of the time window). As a result, to obtain the daily estimation of the number of cases, the cumulative reported estimation provided on a 7-day rolling time window is divided by 7 . After summing this estimation for all the administrative areas belonging to a given sector of the OD matrix, we finally obtain an estimation of the number of COVID-19 cases per sector and per day. COVID-19 data cover the period from November 2, 2020 toMarch 19, 2021.

For our simulation, the number of cases in all sectors is initialized as on November 2, 2020. For the local transmission of the virus (within the sector), we consider the reproduction number $R_{0}=2.5$ Organization W.H.et al. 2020. The infection rate $\beta$ and recovery rate $\mu$ are adjusted according to the value of $R_{0}$. The reported cases in Rhône-Alpes region

${ }^{6}$ https://www.insee.fr/fr/statistiques/4228434.

7 https://www.data.gouv.fr/fr/datasets/donnees-de-laboratoires-infradepartementales-durant-lepidemie-covid-19/.
Table 3 Network statistics

\begin{tabular}{ll}
\hline Network Properties & Value \\
\hline Nodes & 10,000 \\
Edges & 9960 \\
Average degree & 1.992 \\
Edge density & 0.0002 \\
Number of triangles & 390 \\
Average clustering coefficient & 0.0038 \\
Number of components & 1117 \\
Reciprocity & 0 \\
\hline
\end{tabular}

in France as well as the forecast cases using the model are shown in Fig. 9 until March 22, 2021. It can be noticed that the model predicted much higher cases of COVID-19 if no restrictions are introduced $(\alpha=1)$, while we can observe that, for $\alpha=0.5$, the number of actual cases and forecast ones are quite close to each other. To explain this result, it is worth to remind that strong mobility restrictions were reintroduced in France by the end of October $2020,{ }^{8}$ after the first lockdown ended during summer. The new restrictions contributed to keep low the number of COVID-19 infections (Actual). Moreover, it is reasonable to assume that mobility and social interactions were already significantly reduced at the beginning of this second lockdown, with respect to prepandemic behaviors, as a consequence of the first COVID-19 wave and previously imposed restrictive measures. In conclusion, this second case study confirms the applicability of the model to forecast a range of predicted number of cases. The latter can thus help the government and health agencies to understand the impact and introduce proportional interventions to restrict the spread of the epidemic.

\section{Evaluation of complex networks model}

In this section, we first explain our experimental setup, and next, we discuss the results of our simulation conducted using the proposed model for complex networks on synthetic network.

\subsection{Experimental setup}

For the analysis, we created a synthetic network using configuration model Newman 2003, which follows the power law distribution (see Fig. 10) with scale-free exponent (gamma) as 3. In particular, we utilize the

\footnotetext{
8 https://www.vie-publique.fr/en-bref/276947-covid-19-un-2e-confi nement-national-compter-du-29-octobre-minuit.
} 


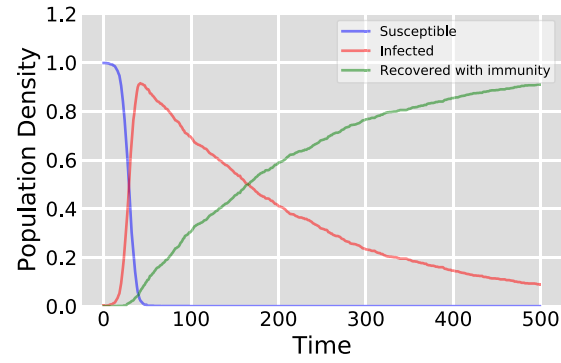

(a) $\alpha=1$

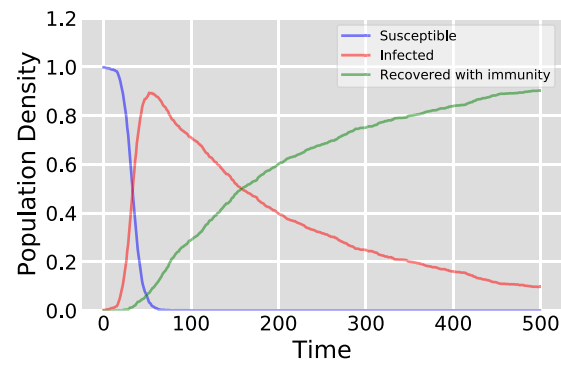

(d) $\alpha=0.7$

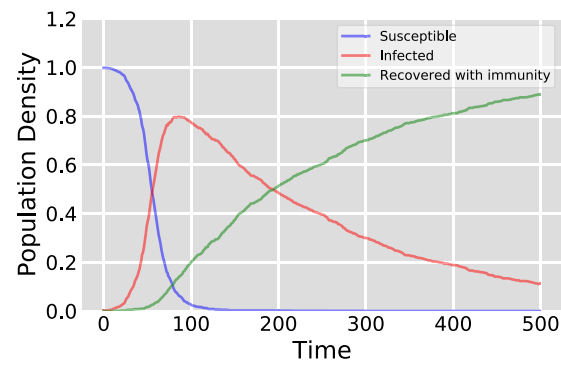

(g) $\alpha=0.4$

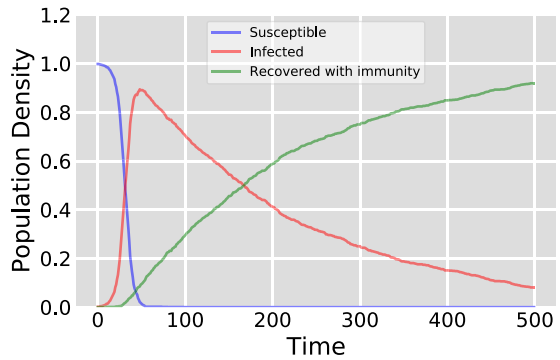

(b) $\alpha=0.9$

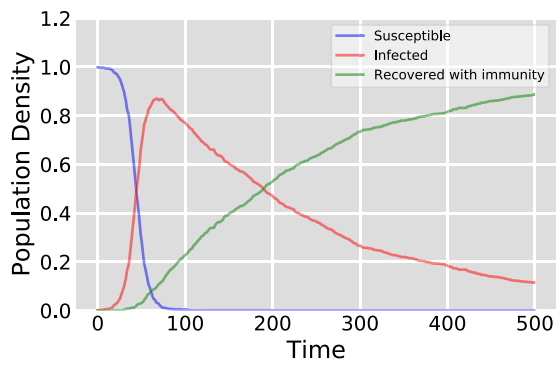

(e) $\alpha=0.6$

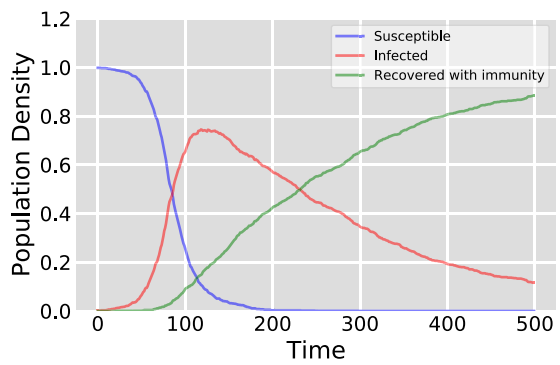

(h) $\alpha=0.3$

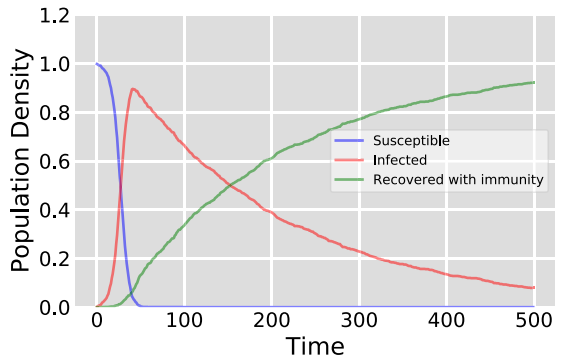

(c) $\alpha=0.8$

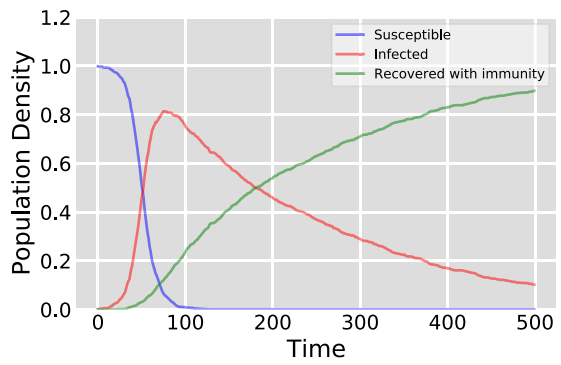

(f) $\alpha=0.5$

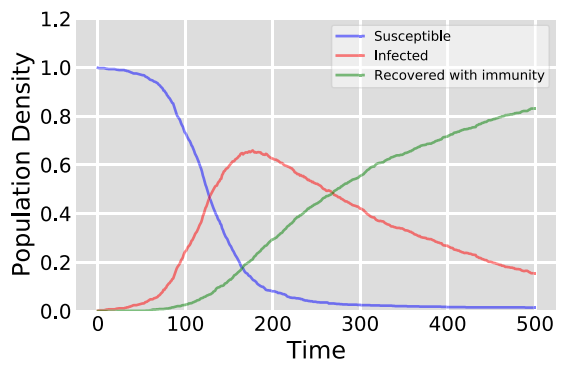

(i) $\alpha=0.2$

Fig. 11 Pandemic origin from random location in complex network: effect of social connectivity parameter ' $\alpha$ '

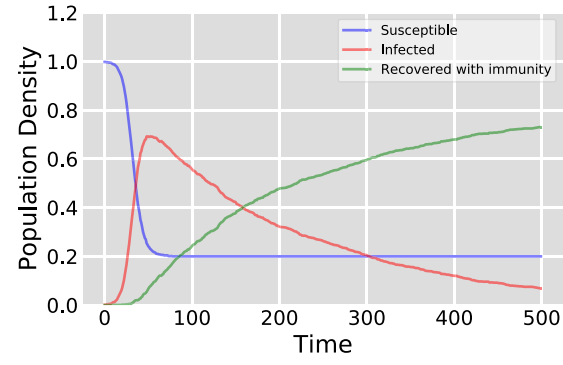

(a) 10\% Locations Quarantine

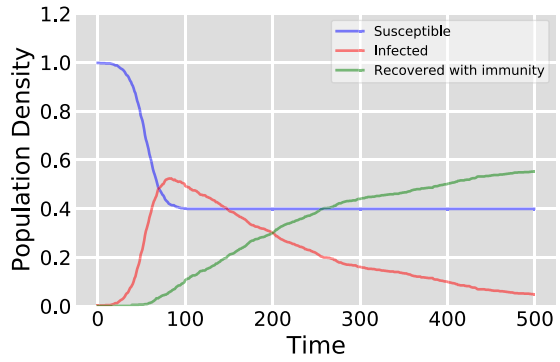

(b) $20 \%$ Locations Quarantine

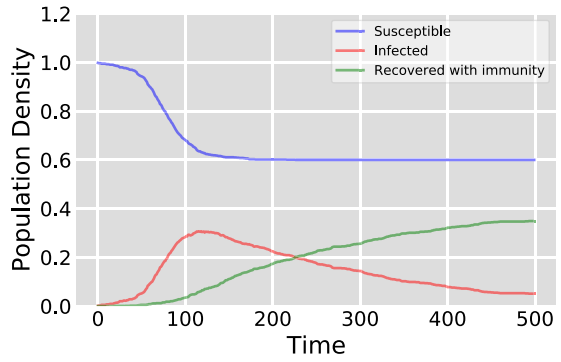

(c) $30 \%$ Locations Quarantine

Fig. 12 Pandemic origin from random location in complex network: effect of quarantine strongly connected locations

random_powerlaw_tree_sequence and configuration model function of networkxHagberg et al. 2008. The configuration model produces a random pseudograph (graph with parallel edges and self-loops) by randomly assigning edges to fit the given degree sequence. We removed all parallel edges and self-loops from our network. Table 3 provides the statistics of our synthetic network. Based on the definition of configuration model and various properties of our synthetic network, we can infer that this network reflects the real-world contact network Voitalov 
et al. 2020. For the rest of this work, we refer to this network as ConNet.

Our simulation is oriented toward addressing the following questions:

- How social connectivity parameter ' $\alpha$ ' affects the fraction of individuals in different compartments (susceptible, infected and recovered) for a complex network?

- What are the outcomes of restricting the mobility (for top-X percentile) of strongly connected locations in a complex network?

\subsection{Results}

We perform various simulation experiments to explain the proposed model for complex network on ConNet by selecting the seed infection location randomly. It is to be noted that the model will behave as a standard SIR model in two cases, (i) if $\alpha=0$, (ii) if the mobility is reduced to 100 percentile (that is no mobility allowed) from connected locations.

Pandemic Origins From a Random Location. Figure 11 displays the influence of the social connectivity parameter ' $\alpha$ ' while keeping the other parameters constant. Figure 11 a to $11 \mathrm{i}$ shows the pandemic dynamics with different values of $\alpha$ starting with $\alpha=1$ to $\alpha=0.2$. We observe that the peak of the infected compartment decreases significantly, as the $\alpha$ decreases, and it also takes longer to reach its peak. This indicates that there is a positive impact of lockdown in controlling a pandemic.

The effect of restricting the mobility from the top-X percentile of highly connected locations with other locations is shown in Fig. 12. Figure 12a, f displays the pandemic dynamics with different percentile of mobility restrictions of highly connected locations starting with 10-30\% (keeping $\alpha=0.5)$. We observe that in the case of a pandemic, restricting the mobility from the top- 10 percentile of highly connected locations can reduce the number of individuals who can get infected to $15-21 \%$. Therefore, quarantine plays a vital role during pandemics.

In order to understand the relationship between $\alpha$ and mobility restriction from strongly connected locations, we performed the numerical simulation of the proposed mean-field equations for complex network. The results are similar to the mean-field equations for non-complex network (see Fig. 4). Therefore, we can infer that the social connectivity parameter ' $\alpha$ ' and mobility both plays an important role during pandemics.

\section{Conclusion}

Classical compartmental epidemic models are unable to describe the spreading pattern of pandemics such as COVID-19 as they do not take into account the effect of social connectivity and mobility in the spreading of the virus. Our proposed mobility-based SIR models for fully mixed and complex networks shows the significance of social connectivity and mobility during pandemics by taking into consideration the local and the global transmission rate of the infection. We have simulated the fully mixed model by considering three different origins of the infection, namely random location, weakly connected location, and strongly connected location. Our simulation shows that limiting the social connectivity reduces and delays the peak of the infected compartment. Our analysis also shows that restricting the mobility from the top-10 percentile of connected locations can reduce the number of infected individuals between 18 and $27 \%$. From the mathematical proof for our proposed models, we obtained that the reproduction number $R_{0}$ directly depends upon social connectivity of individuals, number of connected locations and individuals mobility between locations (and degree of the individual in complex networks model) which is in line with our simulation's results. This indicates that introducing isolation and quarantine is effective in fighting a pandemic crisis. Using the proposed model, we also simulated the real-world scenario by considering the COVID-19 cases in Estonia and Rhône-Alpes region in France. Simulation reveals that the mobility-based SIR model can be helpful to forecast the expected number of cases after some proportion of isolation and quarantine is introduced in the society.

We plan to include various future directions for this work such as by simulating the model using additional temporal networks. Another direction would be to use additional mobility data such as transportation networks for better understanding the pandemic behavior. Importantly, we plan to introduce infection delay and recovery delay simultaneously in our future studies.

Author Contributions Rahul Goel contributed to formal analysis, investigation, validation, visualization, and writing-original draft. Loïc Bonnetain contributed to data creation for Rhône-Alpes region and writing-review and editing. Rajesh Sharma contributed to supervision and writing - review and editing. Angelo Furno contributed to supervision and writing - review and editing.

Funding This research is funded by ERDF via the IT Academy Research Programme, and H2020 framework project, SoBigData++, and CHIST-ERA project SAI. This work has also been supported by the French ANR research project PROMENADE (grant number ANR-18-CE22-0008).

Availability of data and material Our CDR datasets are partly location data and cannot be shared due to privacy concerns. Additionally, despite the fact that the datasets are anonymized at two levels, there is still a small chance that specific persons can be identified. The dataset is owned by the two university laboratories involved in this paper and could be accessible for research purposes after signing an NDA (nondisclosure agreement) and on condition of acceptance by the network 
providers who collected the data. However, in this work, we proposed mobility-based models with mathematical derivations that can be applied to any mobility dataset.

\section{Declaration}

Conflict of interest The authors declare that they have no conflict of interest.

Code availability Not applicable. As this work proposes a mathematical model with derivation.

\section{References}

Albert R, Barabási AL (2002) Statistical mechanics of complex networks. Rev Modern Phys 74(1):47

Anderson RM, May RM (1979) Population biology of infectious diseases: part i. Nature 280(5721):361

Anderson RM, May RM, Anderson B (1992) Infectious diseases of humans: dynamics and control, vol 28. Wiley, Hoboken

Arenas A, Cota W, Gómez-Gardeñes J, Gómez S, Granell C, Matamalas JT, Soriano-Paños D, Steinegger B (2020) Modeling the spatiotemporal epidemic spreading of covid-19 and the impact of mobility and social distancing interventions. Phys Rev X 10(4):041055

Arquam M, Singh A, Sharma R (2018) Modelling and analysis of delayed sir model on complex network. In: International conference on complex networks and their applications. Springer, pp 418-430

Barthélemy M, Barrat A, Pastor-Satorras R, Vespignani A (2005) Dynamical patterns of epidemic outbreaks in complex heterogeneous networks. J Theor Biol 235(2):275-288

Bonabeau E (2002) Agent-based modeling: methods and techniques for simulating human systems. Proc Natl Acad Sci 99(suppl 3):7280-7287

Burke DS, Epstein JM, Cummings DA, Parker JI, Cline KC, Singa RM, Chakravarty S (2006) Individual-based computational modeling of smallpox epidemic control strategies. Acad Emerg Med 13(11):1142-1149

Chinazzi M, Davis JT, Ajelli M, Gioannini C, Litvinova M, Merler S, YPiontti AP, Mu K, Rossi L, Sun K, et al. (2020). The effect of travel restrictions on the spread of the 2019 novel coronavirus (COVID-19) outbreak. Science, 368(6489), 395-400.

CSSE, J. COVID-19 Dashboard by the Center for Systems Science and Engineering (CSSE) at Johns Hopkins University. 2020.

Crooks AT, Hailegiorgis AB (2014) An agent-based modeling approach applied to the spread of cholera. Environ Model Softw 62:164-177

CSSE J (2020) Coronavirus covid-19 global cases by the center for systems science and engineering (csse) at johns hopkins university (jhu)

Centers for Disease Control C, Prevention et al. (2012) First global estimates of $2009 \mathrm{~h} 1 \mathrm{n} 1$ pandemic mortality released by cdc-led collaboration

Epstein JM (2009) Modelling to contain pandemics. Nature 460(7256):687-687

Estonia S (2018) Quarterly bulletin of statistics Estonia. An overview of social and economic developments in Estonia. Statistics Estonia, Tallinn 25
Eubank S, Guclu H, Kumar VA, Marathe MV, Srinivasan A, Toroczkai Z, Wang N (2004) Modelling disease outbreaks in realistic urban social networks. Nature 429(6988): 180-184

Fekih M, Bellemans T, Smoreda Z, Bonnel P, Furno A, Galland S (2020) A data-driven approach for origin-destination matrix construction from cellular network signalling data: a case study of Lyon region (France). Transportation, pp 1-32

Goel R, Sharma R (2020) Mobility based sir model for pandemicswith case study of covid-19. In: 2020 IEEE/ACM international conference on advances in social networks analysis and mining (ASONAM), pp 110-117. https://doi.org/10.1109/ASONA M49781.2020.9381457

Goel R, Sharma R, Aasa A (2021) Understanding gender segregation through call data records: an Estonian case study. PLoS ONE 16(3):e0248212

Goel R, Singh A, Ghanbarnejad F (2019) Modeling competitive marketing strategies in social networks. Phys A Stat Mech Appl 518:50-70

Gojovic MZ, Sander B, Fisman D, Krahn MD, Bauch CT (2009) Modelling mitigation strategies for pandemic (h1n1). CMAJ 181(10):673-680

Hagberg A, Swart P, Chult SD (2008) Exploring network structure, dynamics, and function using networkx. Tech. rep., Los Alamos National Lab.(LANL), Los Alamos, NM (United States)

Hethcote HW (2000) The mathematics of infectious diseases. SIAM Rev 42(4):599-653

Hiir H, Sharma R, Aasa A, Saluveer E (2019) Impact of natural and social events on mobile call data records-an estonian case study. In: International conference on complex networks and their applications, pp 415-426. Springer

Huang X, Clements AC, Williams G, Mengersen K, Tong S, Hu W (2016) Bayesian estimation of the dynamics of pandemic (h1n1) 2009 influenza transmission in queensland: a space-time sir-based model. Environ Res 146:308-314

Hurley M, Jacobs G, Gilbert M (2006) The basic si model. New Directions Teach Learn 2006(106): 11-22

Jin Y, Wang W, Xiao S (2007) An sirs model with a nonlinear incidence rate. Chaos Solitons Fractals 34(5):1482-1497

Kermack WO, McKendrick AG (1927) A contribution to the mathematical theory of epidemics. In: Proceedings of the royal society of London a: mathematical, physical and engineering sciences, vol 115, pp 700-721. The Royal Society

Khalil KM, Abdel-Aziz M, Nazmy TT, Salem ABM (2012) An agentbased modeling for pandemic influenza in Egypt. In: Handbook on decision making, pp 205-218. Springer

Kiskowski MA (2014) A three-scale network model for the early growth dynamics of 2014 west Africa Ebola epidemic. PLoS Curr 6

Li X, Wang X (2006) Controlling the spreading in small-world evolving networks: stability, oscillation, and topology. IEEE Trans Autom Control 51(3):534-540

Liu L (2015) A delayed sir model with general nonlinear incidence rate. Adv Differ Equ 2015(1):329

Moreno Y, Pastor-Satorras R, Vespignani A (2002) Epidemic outbreaks in complex heterogeneous networks. Eur Phys J B-Condens Matter Complex Syst 26(4):521-529

Nåsell I (1996) The quasi-stationary distribution of the closed endemic sis model. Adv Appl Probab 28(3):895-932

Newman ME (2003) The structure and function of complex networks. SIAM Rev 45(2): 167-256

Organization WH, et al. (2009) Pandemic h1n1 2009. Tech. rep, WHO Regional Office for South-East Asia

Organization WH, et al. (2020) Coronavirus disease 2019 (covid-19): situation report 46 
Pastor-Satorras R, Castellano C, Van Mieghem P, Vespignani A (2015) Epidemic processes in complex networks. Rev Modern Phys 87(3):925

Schelling TC (1971) Dynamic models of segregation. J Math Sociol 1(2):143-186

Shi H, Duan Z, Chen G (2008) An sis model with infective medium on complex networks. Phys A Stat Mech Appl 387(8-9):2133-2144

Singh A, Singh YN (22012) Nonlinear spread of rumor and inoculation strategies in the nodes with degree dependent tie strength in complex networks. arXiv:1208.6063

Sun R et al (2006) Cognition and multi-agent interaction: from cognitive modeling to social simulation. Cambridge University Press, Cambridge

Towers S, Geisse KV, Zheng Y, Feng Z (2011) Antiviral treatment for pandemic influenza: assessing potential repercussions using a seasonally forced sir model. J Theor Biol 289:259-268

Tuomisto JT, Yrjölä J, Kolehmainen M, Bonsdorff J, Pekkanen J, Tikkanen T (2020) An agent-based epidemic model reina for covid19 to identify destructive policies. medRxiv
Vespignani A (2012) Modelling dynamical processes in complex sociotechnical systems. Nat Phys 8(1):32

Viboud C, Simonsen L, Chowell G (2016) A generalized-growth model to characterize the early ascending phase of infectious disease outbreaks. Epidemics 15:27-37

Voitalov I, van der Hoorn P, Kitsak M, Papadopoulos F, Krioukov D (2020) Weighted hypersoft configuration model. Phys Rev Res 2(4):043157

Xia C, Wang L, Sun S, Wang J (2012) An sir model with infection delay and propagation vector in complex networks. Nonlinear Dyn 69(3):927-934

Zhang JZ, Wang JJ, Su TX, Jin Z (2010) Analysis of a delayed sir epidemic model. In: 2010 international conference on computational aspects of social networks (CASoN), pp 192-195. IEEE

Publisher's Note Springer Nature remains neutral with regard to jurisdictional claims in published maps and institutional affiliations. 